\title{
Remarkable Symmetries in the Milky Way Disc's Magnetic Field
}

\author{
P. P. Kronberg ${ }^{\mathrm{A}, \mathrm{B}, \mathrm{E}}$ and K. J. Newton-McGee $e^{\mathrm{C}, \mathrm{D}}$ \\ A Department of Physics, University of Toronto, Toronto, M5S 1A7, Canada \\ ${ }^{B}$ IGPP, Los Alamos National Laboratory, M.S. T006, Los Alamos, NM 87545, USA \\ C Sydney Institute for Astronomy, School of Physics, The University of Sydney, \\ NSW 2006, Australia \\ D Australia National Telescope Facility, CSIRO, PO Box 76, Epping, NSW 1710, Australia \\ ${ }^{\mathrm{E}}$ Corresponding author. Email: kronberg@physics.utoronto.ca
}

Received 2010 November 29, accepted 2011 March 4

\begin{abstract}
We apply a new, expanded compilation of extragalactic source Faraday rotation measures (RM) to investigate the broad underlying magnetic structure of the Galactic disk at latitudes $|b| \lesssim 15^{\circ}$ over all longitudes $l$, where our total number of RMs is comparable to those in the combined Canadian Galactic Plane Survey (CGPS) at $|b|<4^{\circ}$ and the Southern Galactic Plane (SGPS) $|b|<1.5^{\circ}$. We report newly revealed, remarkably coherent patterns of $\mathrm{RM}$ at $|b| \lesssim 15^{\circ}$ from $l \sim 270^{\circ}$ to $\sim 90^{\circ}$ and $\mathrm{RM}(l)$ features of unprecedented clarity that replicate in $l$ with opposite sign on opposite sides of the Galactic center. They confirm a highly patterned bisymmetric field structure toward the inner disc, an axisymmetic pattern toward the outer disc, and a very close coupling between the CGPS/SGPS RMs at $|b| \lesssim 3^{\circ}$ ('mid-plane') and our new RMs up to $|b| \sim 15^{\circ}$ ('near-plane'). Our analysis also shows the vertical height of the coherent component of the disc field above the Galactic disc's mid-plane — to be $\sim 1.5 \mathrm{kpc}$ out to $\sim 6 \mathrm{kpc}$ from the Sun. This identifies the approximate height of a transition layer to the halo field structure. We find no RM sign change across the plane within $|b| \sim 15^{\circ}$ in any longitude range. The prevailing disc field pattern and its striking degree of large-scale ordering confirm that our side of the Milky Way has a very organized underlying magnetic structure, for which the inward spiral pitch angle is $5.5^{\circ} \pm 1^{\circ}$ at all $|b|$ up to $\sim 12^{\circ}$ in the inner semicircle of Galactic longitudes. It decreases to $\sim 0^{\circ}$ toward the anticentre.
\end{abstract}

Keywords: magnetic fields — methods: observational — Galaxy: disc — Galaxy: structure — radio continuum: ISM

\section{Introduction}

Early extragalactic radio source (EGRS) Faraday rotation measure (RM) probes of the Milky Way magnetic field by Gardner \& Davies (1966), and the first pulsar RM analyses by Manchester $(1972,1974)$ revealed a prevailing magnetic field in the local spiral arm that is clockwise as viewed from the north Galactic pole. Further study with a 555-source extragalactic RM dataset produced the discovery of a large-scale field reversal in the direction of the Sagittarius - Carina arm (Simard-Normandin \& Kronberg 1979). Subsequent numerical analyses by Simard-Normandin \& Kronberg (1980) found a prevailing local field pointing to $l \sim 75^{\circ}$, a more complex pattern in the north Galactic hemisphere, an anomalously high off-plane, high-RM zone ('Region A'), a $\sim 30^{\circ}$ angular autocorrelation scale of RMs off the plane, and a Galactic magneto-ionic scale height of $\sim 1.8 \mathrm{kpc}$.

Confirmation of a large-scale Sagittarius - Carina field reversal has since come from several EGRS and pulsar RM studies (Rand \& Lyne 1994; Han et al. 1999; Frick et al. 2001; Weisberg et al. 2004). Variants of this model have been proposed by Han et al. (2006) - a bisymmetric model with field reversals at each arm/ interarm interface; Vallée (2008) - a concentric ring model having one field reversal; and Sun et al. (2008) who concluded that a combined axisymmetric and toroidal model best describes the Galactic magnetic field. Men et al. (2008), using pulsar RMs, concluded that neither a concentric ring model nor a bisymmetric or axisymmetric field model alone suffices when attempting to describe the Galactic magnetic field.

The high density of EGRS RMs in the $|b|<4^{\circ}$ Canadian Galactic Plane Survey (CGPS) (Brown, Taylor \& Jackel 2003), and in the $|b|<1.5^{\circ}$ Southern Galactic Plane Survey (SGPS) (Brown et al. 2007) were used to probe both small-scale (Haverkorn et al. 2006), and larger-scale field structures (Brown et al. 2007). The best-fit model of the latter has a clockwise field everywhere except for two reversals, one in the Scutum - Crux arm and the other in the molecular ring at a smaller galactocentric radius of $\approx 3.7 \mathrm{kpc}$. Since this work was completed, Van Eck et al. (2011) extended the previous results of Brown et al. (2003, 2007) above after closing observational gaps in the 'mid-plane' $\left(|b| \lesssim 3^{\circ}\right.$ sources. Also since this work was completed, Taylor et al. (2009) published a very large RM compilation using 


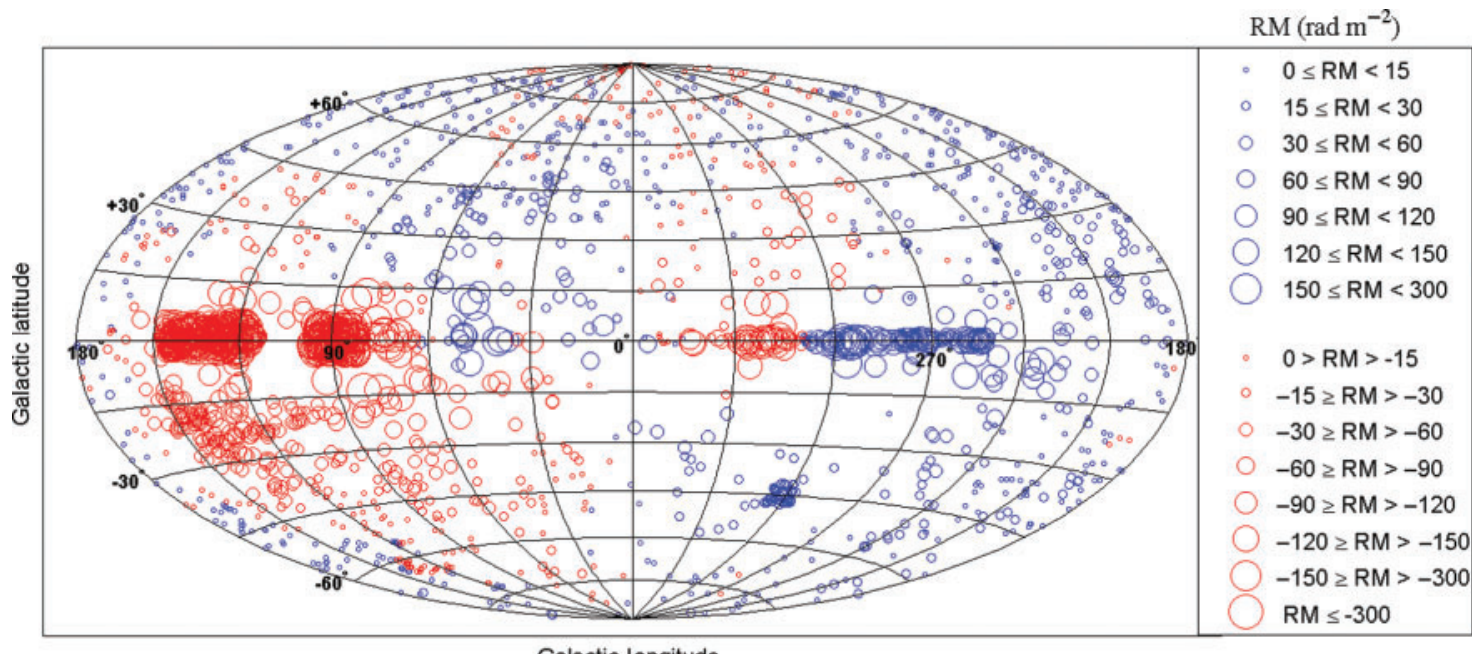

Galactic longitude

Figure 1 An all-sky equal-area projection RM plot of the smoothed RMs from our 2257-source compilation of extragalactic source RMs. The smoothing method is described in the text.

two closely spaced L-band wavelengths in the NVSS survey. However, the NVSS catalogue's zone of avoidance in the southern celestial sphere gives it limited applicability for this investigation since it removes approximately one-third of the $0^{\circ}<|b|<15^{\circ}$ 'nearplane' zone RMs which are the focus this paper.

Conflicting conclusions among some of the above publications illustrate the difficulties in unravelling the magnetic structure of the Galaxy. This paper presents an all-sky plot of the Milky Way's smoothed RM pattern, but focuses solely on the global magnetic structure in the near-plane zone up to $|b| \sim 15^{\circ}$. It uses an improved and expanded set of extragalactic source Faraday RMs of unprecedented average accuracy, most of which were derived from linear polarization measurements over a wide range of wavelengths, from $\lambda \sim 2 \mathrm{~cm}$ to $\lambda \sim 23 \mathrm{~cm}$.

The smoothing 'beam' of $\sim 21^{\circ}$ is comparable to a spiral arm (and inter-arm) width dimension, thereby providing a resolution comparable to some current magnetic field images of nearby external galaxies such as M51. In the 'above-disk' RM sky, the smoothed RM patterns are quite different from those near the disk. Interpretation of the $|b| \gtrsim 12^{\circ} \mathrm{RM}$ sky requires a threedimensional modeling analysis, and this will be the subject of a following paper (Pshirkov et al. 2011).

\section{The Data, Methods and Analysis}

We use a new 2257-RM compilation of $\simeq 1500$ revised and more accurate EGRS RMs of our own (NewtonMcGee \& Kronberg, in prep.), combined with the published CGPS/SGPS RMs, and smaller published lists from Klein et al. (2003), and from Mao et al. (2008). These RM surveys also enable us to explore the match between the 'in-plane' RMs, mostly the CGPS/SPGS data, and the 'near-plane' RMs from our own new data, both above and below the Galactic plane.
Figure 1 shows an equal area, all-sky projection of the smoothed RMs. The RM smoothing method is described in Simard-Normandin \& Kronberg (1980), in which an iterative calculation of the mean RM was performed at each source location, RM $\left(l_{i}, b_{i}\right)$, using all neighbour source RMs within a $15^{\circ}$ radius. At each iteration for a given source, $R M$ values $>1.3 \sigma$ from the mean were eliminated as 'outliers', and a minimum of $4 \mathrm{RMs}$ was required to define a smoothed $\mathrm{RM}$ value at that $(l, b)$. We retested their outlier rejection criterion of $\sim 1.3 \sigma$, using both higher and lower $\sigma$ trial rejection levels, and confirm that it is optimal. The criterion was to minimize the distribution width in $|\mathrm{RM}|$ of Galaxycorrected RMs using only sources at high $|b|$ where the Galactic contribution to RM is smallest compared to the source-to-source RM scatter.

In the southern Galactic plane from $l \approx 270^{\circ}$ to $l \approx$ $355^{\circ}$, one major RM sign change occurs at our smoothing resolution, at $l \approx 310^{\circ}$ (Figure 2 (a)). We note that changes of RM sign on smaller scales have been found in the 'midplane' zone by Brown et al. (2007), and Van Eck et al. (2011). Our smaller number of sign changes is purely an effect of our smoothing half-width, $\approx 21^{\circ}$, which averages over some (real) smaller-scale RM sign changes. This also applies to other $l$-zones near $b=0^{\circ}$, and at some higher latitudes that we do not discuss in this paper. Our method effectively isolates larger-scale RM features in the vicinity of the Milky Way disc, as they would be seen by an extragalactic observer.

The analysis that follows focuses on $|b| \lesssim 15^{\circ}$. It imposes cuts of the RM data variously, and as discussed below, at $|b|=2^{\circ}, 5^{\circ}, 10^{\circ}, 12^{\circ}$, - and finally $20^{\circ}$ to explore the outer $|b|$ limits of the disc-associated field (Section 3.3). Putting these angles in the context of disc $z$-heights, an RM sightline at $|b|=10^{\circ}$ corresponds to a $1.4 \mathrm{kpc} z$-height in the Milky Way disk at $8 \mathrm{kpc}$ from the Sun. This reference value of $1.4 \mathrm{kpc}$ is similar to several estimates of the disc magneto-ionic scale height (e.g., 
(a)

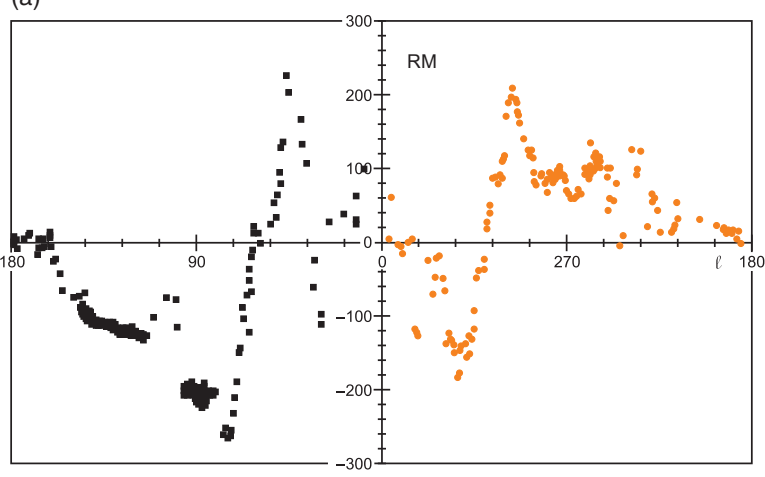

(b)

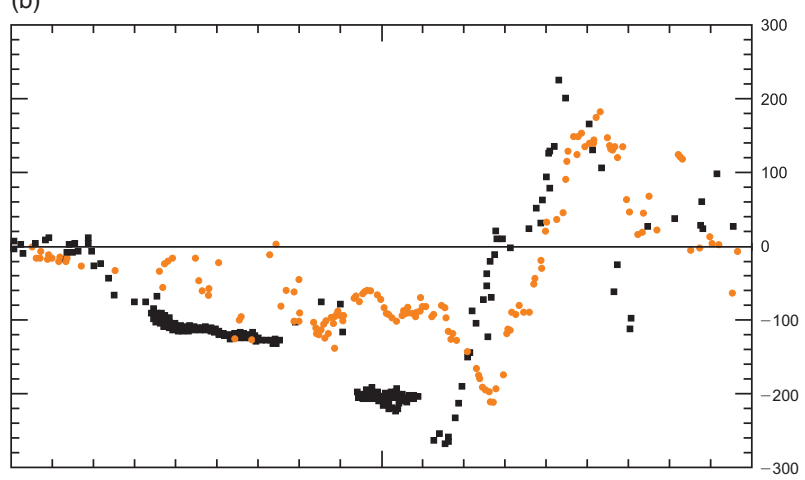

(c)

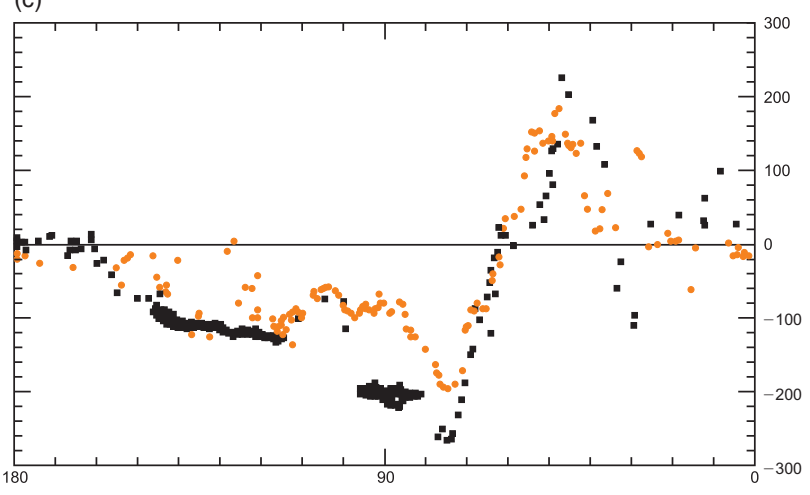

Figure 2 Panel (a): Plot of all smoothed RMs having $|b|<10^{\circ}$ versus $l$. Panel (b): Smoothed RMs with reversed sign and 'flipped' about $l=0^{\circ}$. Panel (c): As in (b) except 'flipped' about $l=349^{\circ}$ $\left(-11^{\circ}\right)$.

Simard-Normandin \& Kronberg 1980 and more recent analyses). It is also comparable to the galaxy's synchrotron radiation thick disc height of $\sim 1.2-1.9 \mathrm{kpc}$ (Beuermann et al. 1985), and to the ionized gas $z$-height estimate (Gaensler et al. 2008). The following analysis indicates clear large-scale patterns and symmetries on our side of the Milky Way disc that were either not previously apparent, or as clear.

\section{Highlights of the Disc Magnetic Field Analysis}

\subsection{Determination of the Prevailing Magnetic Field Direction on Our Side of the Galaxy}

The smoothed RMs for $|b|<10^{\circ}$ are shown in Figure 2(a). The second Galactic $l$-semicircle is then folded, at $l=0^{\circ}$, onto the first. When we then reverse the RM signs in this folded semi-circle (Figure 2(b)) a clear symmetrical and anti-symmetrical pattern emerges in the smoothed RMs. Specifically, the forms of the smoothed or averaged RMs are strikingly similar, but they have opposite signs on opposite sides of the Galactic center.

Figure 2(b) also shows clearly that the Galactic centre direction, $l=0^{\circ}$, is not the correct symmetry axis. An $11^{\circ}$ relative shift of the folded datasets needs to be applied i.e., fold, invert sign, and shift, in that order. This same order is followed throughout the paper. For optimum determination of this shift, we examined RMs between $44^{\circ}$ to $76^{\circ}$ of the post-shift folding centre, and applied a least squares minimization of the residual as a function of shift angle. Figure 2(c) shows the remarkably good overlap that results. This shift corresponds to a pointing of the prevailing local $B$-field to $l=84.5 \pm 1^{\circ}$, i.e., inward from the $l=90^{\circ}$ tangential by $5.5 \pm 1^{\circ}$.

Independently, when we examine the all-sky RM map in Figure 1 at higher latitudes near $l=180^{\circ}$, a similar change from negative to positive RMs is evident near the anti-centre direction nearly all the way to the South Galactic Pole. Above the plane, a similar negative-topositive RM 'border' can be traced as far as $b \simeq+45^{\circ}$, where it then follows a different sign boundary near to the $b=45^{\circ}$ line. We do not attempt to interpret this latter northern high-latitude wandering here, except to note that Frick et al. (2001) attribute the north - south pattern asymmetry at higher $|b|$ to either a stronger field in the southern Galactic hemisphere or to the Sun being located close to the top of a magnetic loop.

The $l=84.5 \pm 1^{\circ}$ magnetic direction is very close to the value of $l=82.8 \pm 4.1^{\circ}$ for local field lines derived from starlight polarizations by Heiles (1996), after correcting for larger-scale spiral arm curvature. In general, any magnetic pitch angle determination will slightly depend on precisely what distance range from the Sun is defined as local. Since our Galactic disk RMs represent typical pathlengths up to several kpc through the disc (see Section 2), this remarkably close agreement between the present analysis and the Galactic curvature-corrected starlight polarization results may be partly coincidental. The agreement with completely independent starlight polarization results is nonetheless worth noting.

The patterns seen in Figure 2 indicate that an extragalactic observer would see at least our side of the Milky Way disc with a very patterned and organized magnetic spiral structure, with a local pitch angle of $5.5^{\circ}$ near the location of the Sun. This, combined with the symmetrical and antisymmetrical RM patterns seen in Figure 2, strongly indicates that the Milky Way disc is laid out like other 'magnetically organized' spiral galaxies.

\subsection{A Test for 'Magnetic Coupling' Between the Mid-Plane Disc and the Immediate 'Above-Plane' Zones}

We now repeat the procedures in Figure 2, using sources having $|b|<10^{\circ}$, but omit the RMs at the very low 


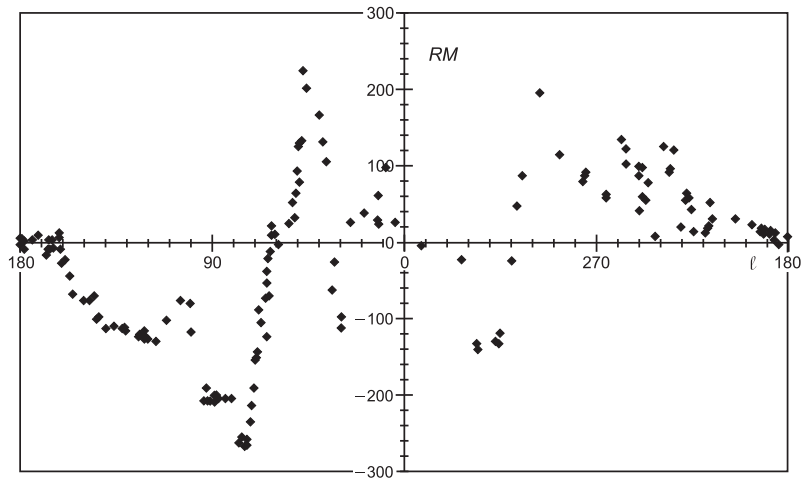

Figure 3 Plot of the smoothed RMs around the Galactic plane as in Figure 2(a), but excluding all the CGPS and SGPS sources, and all other sources at the lowest Galactic latitudes below $|b|=2^{\circ}$.

latitudes $\left(|b|<2^{\circ}\right)$, i.e., close to the Galactic mid-plane. We also exclude any CGPS RMs that are found up to $|b|=4^{\circ}$. This leaves $210 \mathrm{RMs}$ from our compilation at $2^{\circ}<|b|<10^{\circ}$, and these are spread around the entire $l$ circle. As mentioned above, all the $(l, b)$ subset exclusions were done before re-smoothing.

Figure 3 shows patterns that are virtually identical with Figure 2(a), including the crossover points. Application of our fold-reverse-shift-optimize technique in Figure 2(b) and (c) yields the same best-fit spiral pitch angle, $l_{0}=84.5^{\circ} \pm 1^{\circ}$, as when the $|b|<2^{\circ}$ RMs are included. The same result (not shown) is also obtained for the ' $|b|<2^{\circ}$ only' subset of RMs. This result is notable for two reasons. (1) The close agreement occurs between two key non-overlapping datasets - our source sample above $|b|=2^{\circ}$, and the CGPS/SGPS RMs at the lowest $|b|$ s. This also provides a convincing posterior check on the reliability of both RM data sets. (2) Astrophysically, it demonstrates a close coupling between the magnetic field structure in the ISM of the in-plane zone, and at $|z|$-heights up to $\sim 1 \mathrm{kpc}$ away from the mid-plane.

In the above we did not distinguish RM data above and below the plane. We next test for magnetic sign asymmetries and antisymmetries across the Galactic plane. As in Figure 3, we omit all RMs at $|b|<2^{\circ}$. To have adequate statistics for this purpose, we slightly extend the upper boundaries in $|b|$ to $\pm 12^{\circ}$. Then we re-apply the foldreverse-shift-optimize technique separately for RMs at $-12^{\circ}<b<-2^{\circ}$ and $+12^{\circ}>b>+2^{\circ}$.

The same best fit procedure for the prevailing Galactic $B$-field direction gives $l_{0}=84.5 \pm 2^{\circ}$ in the negative $b$ range, and $l_{0}=86.5 \pm 2^{\circ}$ in the positive $b$ range. The magnetic field patterns in $l$ are therefore the same within the error limits, above and below the plane, $84.5^{\circ}$ (or $11^{\circ}$ relative shift after folding). They also agree with the 'fullplane' results in Figure 2.

The respective plots are combined in Figure 4. Here we see that, at our $\sim 21^{\circ}$ smoothing resolution, the sign of the prevailing disc magnetic field pattern does not change through the mid-plane. More explicitly, within the longitude boundaries $l \sim 280^{\circ}$ to $\sim 60^{\circ}$ (the 'inner' longitude

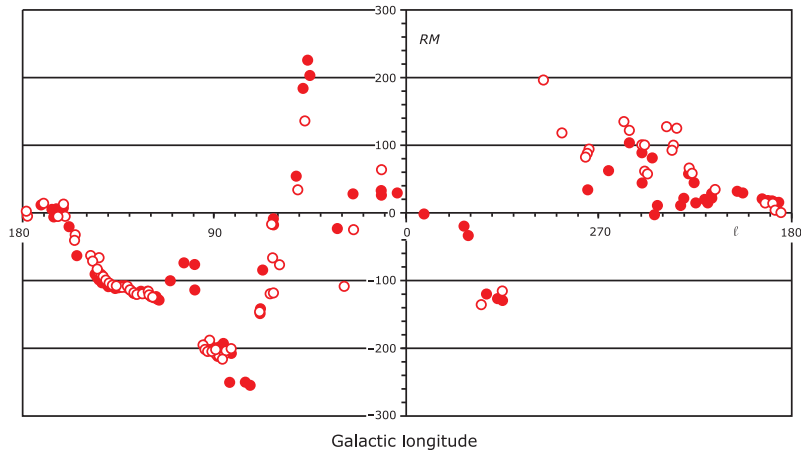

Figure 4 Separate plots of the smoothed RMs around the Galactic plane, splitting by negative $b$ (open circles) and positive $b$ (filled circles), and extending the upper $|b|$ limit of data selection to $|12|^{\circ}$. It demonstrates the consistency of the prevailing magnetic field sign across the Galactic disc.

range) where the RM signs change with $l$, these changes replicate locally with even symmetry, i.e., $\mathrm{RM}(+b) \simeq$ $\mathrm{RM}(-b)$.

It is important here to emphasise that, at these inner longitudes, the even RM sign symmetry across the midplane just demonstrated in Figure 4 holds only at latitudes within the $|b| \lesssim 15^{\circ}$ zone of the galactic disk that that we have been discussing. At some 'inner' longitudes, sign changes in certain $l$ ranges do occur beyond $|b| \sim 15^{\circ}$. This can be seen in Figure 1, and partially seen in the (un-blanked portion of the) RM distribution of Taylor et al. (2009). The nearest approximation to our crossplane sign symmetry result was obtained by Frick et al. (2001) in their wavelet analysis of the 555 RM dataset of Simard-Normandin et al. (1981). Our new combination of the CGPS and SGPS plus the $|b| \lesssim 15^{\circ}$ subset of our new 1500 RMs can now more precisely clarify how, and at what off-plane latitudes, systematic changes in RM sign with $b$ do occur. The RM sign symmetry in $b$ qualitatively confirms the conclusion of Frick et al. (2001), but contrasts with the conclusion of Jansson et al. (2009) on RM sign antisymmetry with respect to $b$. However, RM antisymmetry does occur at some longitudes if all RMs at $|b|<15^{\circ}$ are blanked out.

Outward of $l \sim 280^{\circ}\left(l<280^{\circ}\right)$ and of $l \gtrsim 60^{\circ}$, the Galactic field has the same prevailing sign above, in, and below the Galactic plane. This includes the 'in-plane' $\mathrm{RM}$ regions of the CGPS and SGPS $\left(|b|<4^{\circ}\right.$ and $<1.5^{\circ}$, respectively). Below the plane, the RMs keep the same prevailing sign at all $l$ ranges from $l \gtrsim 60^{\circ}$ to $l \lesssim 330^{\circ}$ right down to the South Galactic Pole. These results confirm that the symmetry of 'outer longitude' RMs with respect to the mid-plane is consistent with an overall quadrupole (even) symmetry of the large scale field. This question is further investigated in a following paper that analyses the entire $|b|>10^{\circ} \mathrm{RM}$ data of Figure 1 (Pshirkov et al. 2011).

Note the general point of distinguishing outer l's (i.e., toward the anticentre) from true radial distances from the Sun to the outer Galaxy. 


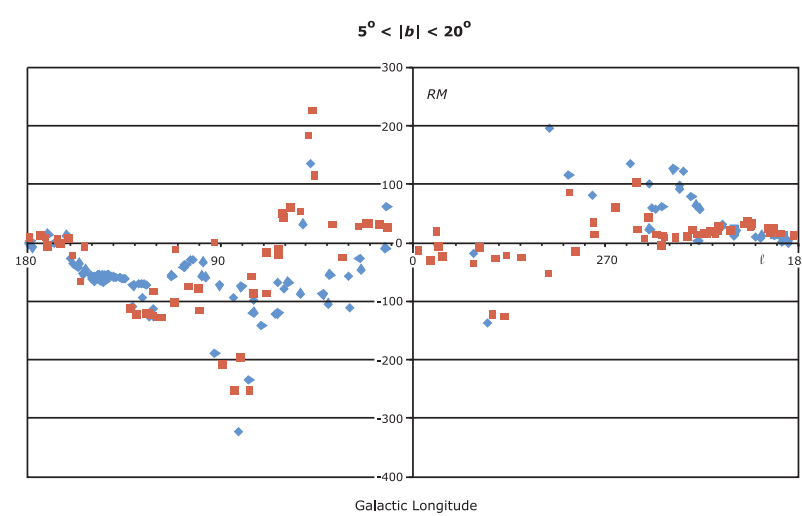

Figure 5 Plot of the smoothed RMs as in Figures 3 and 4, but including only RMs at a higher range of $20^{\circ}>|b|>5^{\circ}$. Points above the plane (diamond symbols) and below it (square symbols) are distinguished.

\subsection{A New Test for the z-Extent of the Regular Disc Field}

Finally, we revisit evidence for the thickness of the magnetic field coherence zone in our vicinity of the Milky Way disc. To do this, we raise the upper $|b|$ boundary further from the plane, recalling that $|b|=10^{\circ}$ corresponds to a $1.4 \mathrm{kpc} z$-height at $8 \mathrm{kpc}$ distance (approximately the distance to the Galactic centre). Our RM smoothing resolution at this distance is close to optimal for this purpose. To put our RM smoothing 'beam' in a wider scale context: at $6 \mathrm{kpc}$ distance our 'beam' is roughly comparable to (a) the full width of the spiral arm ionized gas; (b) a spiral arm width in HI in the Galactic $(x, y)$ plane; and (c) approximately an inter-arm separation.

As the selection boundaries of $|b|$ increase away from $|b| \sim 10^{\circ}$, the sharp features and symmetries seen in Figures 2 and 3 become progressively less distinct. Figure 5 shows all smoothed RM points having individual RMs pre-selected to $5^{\circ}<|b|<20^{\circ}$. While the distinctive shapes in Figures 2 and 3 can still be recognised, they are no longer as pronounced. That is, our fold-reverse-shift method no longer produces a clear merging of patterns, and the underlying spiral pattern 'fades away' as we proceed toward the Galactic halo. In this way we have established the approximate transition zone between the Milky Way's magnetic disk and halo, which is in the range 1-2 kpc above the Galactic mid-plane. The location of this transition agrees with the conclusion of Jansson et al. (2009), based on a combination of the WMAP $22 \mathrm{GHz}$ data and a smaller set of RMs.

\section{Summary}

We have discussed new and striking underlying magnetic field patterns in the Galactic disc by suitably analysing an expanded and revised compilation of 2257 extragalactic RMs. The unprecedented clarity of our results comes from: (1) the increased numbers, and wide $\lambda^{2}$ range used for RMs immediately around the Galactic plane; and (2) our chosen smoothing scale, corresponding to $z$ $\sim 1 \mathrm{kpc}$ at a distance of $6 \mathrm{kpc}$, preferentially detects features on a spiral arm and inter-arm scale up to $\sim 8 \mathrm{kpc}$ from the Sun. Our analysis is thus sensitive to the largescale underlying magnetic geometry of the Galactic disc and favours longer Galactic path lengths.

The RM variations in a band from $b=+10^{\circ}$ to $-10^{\circ}$ about $l=0^{\circ}$ around the Galactic plane show clear and sharp sign changes. There is striking mirror symmetry about a reference direction $l=-5.5^{\circ}$. This gives a precisely determined, $l=84.5 \pm 1^{\circ}$ (i.e., $l=90-5.5^{\circ}$ ), mean magnetic field direction in the vicinity of the Sun - more precisely determined than in earlier analyses. In a threedimensional representation, this inward spiral tilt occurs over the approximate $\sim 1.5 \mathrm{kpc} z$-height of the Milky Way disc, at least in our broad Galactic vicinity. We clearly rule out a circular magnetic field pattern near our Galactic radius. The distinct 'softening' of the $\mathrm{RM}(l)$ patterns at $|b| \gtrsim 15^{\circ}$ in Figure 5 indicates that these global patterns are confined to $z$-heights within $1-2 \mathrm{kpc}$ of the Milky Way's disc, and that other magnetic patterns emerge as we proceed further from the Galactic plane into the halo.

We have shown in Section 3.2 that the prevailing magnetic field direction does not change sign across the galactic disc, i.e., that the magnetic field sense is even with respect to $z=0$. This applies at all $l$ ranges at $|b|<12^{\circ}$, i.e., where $\langle\mathrm{B}\rangle$ changes sign with $l$, the same sign is locally preserved on the opposite side of the Galactic plane. It is consistent with, but does not uniquely confirm, the quadrupole symmetry predicted by dynamo theory (e.g., Vallée 1992).

The improved definition of the magnetic structure of the Galactic disc is of interest for understanding and comparing the global magnetic structure of the Milky Way and other galaxies, and their evolution (e.g., Beck et al. 1997; Shukurov et al. 2006), and for understanding the propagation of charged, very-high-energy cosmic ray nuclei through our Galaxy (e.g., Auger Collaboration 2007).

\section{Acknowledgments}

The principal results of this paper form part of a Ph.D. thesis completed in early 2009 (K.N.M.), and appeared, in part, in arXiv:0909.4753. P.P.K. acknowledges support from an NSERC (Canada), the U.S. DOE (LANL), the hospitality of CSIRO, and Prof. Bryan Gaensler at the University of Sydney. P.P.K. and K.N.M. gratefully acknowledge support from the Australian Research Council Federation Fellowships of Prof. Bryan Gaensler (FF0561298) and Prof. Ron Ekers (FF0345330) respectively. We are grateful to the anonymous referee for helpful comments.

\section{References}

Auger Collaboration, 2007, Science, 318, 938

Beck, R., Brandenburg, A., Moss, D., Shukurov, A. \& Sokoloff, D. D., 1997, ARAA, 34, 155

Beuermann, K., Kanbach, G. \& Berkhuijsen, E. M., 1985, A\&A, 153,17

Brown, J. C., Haverkorn, M., Gaensler, B. M., Taylor, A. R., Bizunok, N. S., McClure-Griffiths, N. M., Dickey, J. M. \& Green, A. J., 2007, ApJ, 663, 238 
Brown, J. C., Taylor, A. R. \& Jackel, B. J., 2003, ApJS, 145, 213 Frick, P., Stepanov, R., Shukurov, A. \& Sokoloff, D., 2001, MNRAS, 325, 649

Gaensler, B. M., Madsen, G. J., Chatterjee, S. \& Mao, S. A., 2008, PASA, 25, 184

Haverkorn, M., Gaensler, B. M., Brown, J. C., Bizunok, N. S., McClure-Griffiths, N. M., Dickey, J. M. \& Green, A. J., 2006, ApJL, 637, L33

Han, J. L., Manchester, R. N. \& Qiao, G. J., 1999, MNRAS, 306, 371

Han, J. L., Manchester, R. N. \& Qiao, G. J., 2006, ApJ, 642, 868

Heiles, C., 1996, ApJ, 462, 43

Jansson, R., Farrar, G. R., Waelkens, A. H. \& Enßlin, T. E., 2009, J. Cosm. \& Particle Physics, 7, 21

Klein, U., Mack, K.-H., Gregorini, L. \& Vigotti, M., 2003, A\&A, 406, 579

Manchester, R. N., 1972, ApJ, 172, 43

Manchester, R. N., 1974, ApJ, 188, 637

Mao, S. A., Gaensler, B. M., Stanimirovic, S., Haverkorn, M., McClure-Griffiths, N. M., Staveley-Smith, L. \& Dickey, J. M., 2008, ApJ, 688, 1029
Men, H., Ferrière, K. \& Han, J. L., 2008, A\&A, 486, 819

Pshirkov, M. S., Tinyakov, P. G., Kronberg, P. P. \& NewtonMcGee, K. J., 2011, ApJ (submitted)

Shukurov, A., Sokoloff, D., Subramanian, K. \& Brandenburg, A., 2006, A\&A, 448, L33

Sun, X. H., Reich, W., Waelkens, A. \& Enßlin, T. A., 2008, A\&A, 477,573

Simard-Normandin, M. \& Kronberg, P. P., 1979, Nature, 279, 115

Simard-Normandin, M. \& Kronberg, P. P., 1980, ApJ, 242, 74

Simard-Normandin, M., Kronberg, P. P. \& Button, S., 1981, ApJS, 45, 97

Taylor, A. R., Stil, J. M. \& Sunstrum, C., 2009, ApJ, 702, 1230

Vallée, J. P., 2008, ApJ, 681, 303

Vallée, J. P., 1992, A\&A, 255, 100

Van Eck, C. L., Brown, J. C., Stil, J. M., Rae, K., Gaensler, B. M., Shukurov, A., Taylor, A. R., Haverkorn, M., Kronberg, P. P. \& McClure-Griffiths, N. M., 2011, ApJ, 728, 97

Weisberg, J. M., Cordes, J. M., Kuan, B., Devine, K. E., Green, J. T. \& Backer, D. C., 2004, ApJS, 150, 317 